\title{
Jan Engberg
}

\section{Radegundis Stolze: Hermeneutisches Übersetzen. Lin-} guistische Kategorien des Verstehens und Formulierens beim Übersetzen (= Tübinger Beiträge zur Linguistik, 368). Tübingen: Gunter Narr Verlag, 1992.

Die Arbeit von Radegundis Stolze besteht aus einem theoretischen und einem praktischen Teil. Im theoretischen Teil (13-88) stellt sie ihre Theorie zum Übersetzen als humanbestimmte Aktivität, die durch Hermeneutik am besten beschrieben werden kann, dar. Sie stellt dabei fünf translatorische Kategorien auf. Diese Kategorien sind Teilgebiete der Sprachwissenschaft (THEMATIK, SEMANTIK, LEXIK, PRAGMATIK und STILISTIK). Die Anwendung der Kategorien bei der Beschreibung des Übersetzungsprozesses und bei der Übersetzungskritik wird im praktischen Teil dargestellt (89-276). Der Anhang (277-388) enthält 18 authentische Texte und Übersetzungsvorschläge zu diesen Texten, die als Beispielmaterial für den praktischen Teil dienen, sowie eine ausführliche Bibliographie.

Radegundis Stolze sieht die Übersetzungswissenschaft als eine Kommunikationswissenschaft außerhalb der Linguistik an. Das Übersetzen kann auf drei Ebenen beschrieben werden. Situationell hat der Übersetzer die Rolle eines Sprachmittlers, der die Verständigung zwischen Kommunikationspartnern aus verschiedenen Kulturen ermöglichen soll. Handlungspraktisch ist das Übersetzen das Übertragen einer Mitteilung in eine andere Sprache, und sprachwissenschaftlich ist es das Verstehen und Neuformulieren eines sprachlich gestalteten Textes. Übersetzen ist somit nicht das Übertragen von ausgangssprachlichen in zielsprachliche Textstrukturen nach festlegbaren Regeln, sondern verstehender und neuformulierender Umgang mit Texten. Nach Stolze ist die Sprachwissenschaft eine Hilfsdisziplin der Übersetzungswissenschaft. Nach unserer Auffassung ist eine solche Unterscheidung etwas zu streng. Genau wie die Text-linguistik ist die Übersetzungswissenschaft eine Disziplin, die mehrere Zweige der Linguistik integrieren muß, um ihren Gegenstand adäquat beschreiben zu können. Dadurch plaziert sie sich aber nicht außerhalb der Sprachwissenschaft, denn alle drei Beschreibungsebenen, 
die Stolze angibt, gehören in unserer Sicht zum Gegenstand dieser Wissenschaft.

Da also nach Stolze als Ausgangspunkt nicht die ausgangstextlichen Strukturen als solche, sondern nur der ganzheitliche Sinn der Textvorlage (Ausgangstext) der Übersetzung (Zieltext) Bindungen auferlegt, hat der Übersetzer bei der Formulierung der Übersetzung eine weitgedehnte Freiheit. Es gibt aber Beschränkungen dieser Freiheit. Primär bestehen sie darin, daß die Übersetzung die Mitteilung (den Sinn) der Vorlage loyal, möglichst detailliert und intentionsadäquat wiedergeben soll. Unter "Sinn" wird das im Text Gemeinte verstanden, wie es aus dem Gesagten und den Äußerungsbedingungen erschließbar ist. Dabei können Normen und Konventionen der zielsprachlichen Textsorte oder kulturgebundene Formulierungsweisen eine Rolle spielen. Der Übersetzer muß also bei der Neuformulierung Auswahlentscheidungen treffen. Es ist Aufgabe der Übersetzungswissenschaft, die für solche Entscheidungen notwendige Fähigkeit (Übersetzungskompetenz) zu beschreiben.

In der Theorie von Stolze spielen die Begriffe "Intuition" und "Stimmigkeit" eine große Rolle. Intuition wird als wesentlicher Teil des menschlichen Verstehens und somit als notwendiger Teil des Über-setzungsprozesses gesehen. Anhand des intuitiven Verständnisses der Textvorlage, das auf sein sprachliches und sonstiges Vorwissen baut, fertigt der Übersetzer einen Übersetzungsvorschlag an, der dann durch rationale Überlegungen nachgeprüft und gegebenenfalls geändert wird. Die rationalen Überlegungen können durch die obengenannten translatorischen Kategorien gesteuert werden. Intuition und damit verbunden auch Kreativität gehören also zu den bei jeder Übersetzung einzusetzenden Denkmethoden. Mit der Einschränkung, daß in vielen Fällen der nicht-literarischen Übersetzung Textsortenkonventionen und andere Faktoren die Kreativität begrenzen, halten wir diese Auffassung der Rolle der Intui-tion im Übersetzungsprozeß für möglich. Ob sie richtig ist, können nur kognitionspsychologische Studien zeigen.

Als Maßstab dafür, wann eine Übersetzung mit einer Textvorlage übereinstimmt, entwirft Stolze in Anlehnung an den Symmetrie-Begriff der naturwissenschaftlichen Evolutionsforschung einen graduellen Begriff der Stimmigkeit, der den Begriff der Äquivalenz ersetzen soll. Den äquivalenz-Begriff lehnt sie ab, weil er zu stark ergebnis- und zu wenig prozeßorientiert ist. Sie faßt den Begriff der Äquivalenz als ein Instrument der Übersetzungskritik an fertigen Übersetzungen auf, wogegen die Stimmigkeit ein Parameter bei der Bearbeitung eines Überset- 
zungsvorschlages ist. Mit dem höchsten Grad der Stimmigkeit ist die Sinneinheit von Textvorlage und Übersetzung gemeint. Die Stimmigkeit ist erreicht, "wenn das Gemeinte mühelos beim Leser ankommt" (72). Die Begriffe Intuition und Stimmigkeit sind in der Theorie verbunden. Der auf der Basis der Intuition angefertigte Übersetzungsvorschlag wird solange überarbeitet, bis der gewollte oder in der gegebenen Situation mögliche Stimmigkeitsgrad erreicht ist.

Unseres Erachtens ist der Begriff der Stimmigkeit ein mögliches Mittel zur Beschreibung des Übersetzungsprozesses. Er zeichnet sich gegenüber dem ähnlichen, von Reiß/Vermeer (1984) vertretenen Begriff der Adäquatheit dadurch aus, daß er die Übereinstimmung zwischen Textvorlage und Übersetzung bezüglich des Sinns der Texte betont. Jedoch kommt die Einbeziehung der Funktion der Übersetzung bei der Entscheidung der Stimmigkeit theoretisch gesehen zu kurz. Zwar wird angeführt, daß "bei unterschiedlicher Gewichtung von Übersetzerentscheidungen [...] auch verschiedene Übersetzungen desselben Textes jeweils eine optimale Stimmigkeit zur Textvorlage erzielen [können]" (72). Es wird aber nicht weiter ausgeführt, wodurch die unterschiedliche Gewichtung verursacht wird. In einem anderen Zusammenhang sagt Stolze, daß die Stimmigkeit darin besteht, daß "beide Texte miteinander das Ganze haben" (82). Und in Verbindung mit einer späteren Analyse sagt sie, daß die Relation der Stimmigkeit impliziert, daß "möglichst viele ihrer Qualitäten (d.h. der Textvorlage, Anm. d. Verf.) in der Zielsprache wiederkehren" (258).

Eine Auswahl von Stimmigkeitsgebieten je nach Funktion der Übersetzung scheint sie in den letztgenannten Beispielen somit nicht akzeptieren zu wollen, obwohl sie am Anfang des Kapitels bezüglich der Übereinstimmung von Textvorlage und Übersetzung folgendes Gebot für Übersetzer formuliert hat: "Der Übersetzer soll die verstandene Mitteilung so neu zur Sprache bringen, daß eine optimale Sinnübereinstimmung zwischen Text und Übersetzung entsteht, welche zugleich ihren Zweck er- füllt" (61). Um wirklich anwendbar zu werden, muß der Begriff der Stimmigkeit die Funktionsadäquatheit der Übersetzung und auch die Möglichkeit der Übertragung von Teilsinn stärker berücksichtigen, um die Multiperspektivität von Texten adäquat beschreiben zu kön-

1 Gutt (1991) stellt ein Konzept dar, das einer Erklärung von Stimmigkeit näher kommt. Mit dem Begriff der "Communicative Clues" beschreibt er die Relation zwischen dem Sinn eines Textes und seinen Strukturelementen. Je nach Übersetzungstyp können Teile des Sinns und dadurch auch Teile der Struktur der Textvorlage berücksichtigt und übernommen werden. Für eine nähere Diskussion des Ansatzes siehe Engberg (1992). 
nen ${ }^{1}$.

Im praktischen Teil der Arbeit hat Radegundis Stolze die lobenswerte Anstrengung auf sich genommen und gewagt, ihre Theorie ausführlich an Übersetzungsbeispielen vorzuführen. Es werden die fünf translatorischen Kategorien THEMATIK, SEMANTIK, LEXIK, PRAGMATIK und STILISTIK vorgestellt und ihre Beschreibungseffizienz an Beispielen getestet. Stolze postuliert, daß die ersten drei Kategorien primär zur Rezeptions-, die letzten beiden Kategorien primär zur Produktionsphase des Übersetzungsprozesses gehören. Die angeführten Beispiele zeigen aber unseres Erachtens mit aller Deutlichkeit, daß eine solche Einteilung jedenfalls generell nicht haltbar ist. Die Kategorien sind interdependent, und die dazugehörigen Untersuchungsmethoden müssen folglich sowohl bei der Rezeption als auch bei der Produktion eingesetzt werden. So werden bei der Kategorie THEMATIK solche Punkte wie die Ermittlung der Entstehungssituation (darunter, ob ein Text ein Fachtext ist) und die Einbeziehung von Kulturspezifika erwähnt, die später bei der Präsentation der Kategorie PRAGMATIK wiederkehren. Auch bei der Kategorie SEMANTIK wird PRAGMATIK und STILISTIK notwendigerweise miteinbezogen. Dadurch soll aber den Kategorien ihre Relevanz für die Übersetzungspraxis nicht abgestritten werden. Nur der Zuschreibung von einzelnen Kategorien zu einzelnen Phasen gegenüber sind wir skeptisch.

Die Kategorie THEMATIK umfaßt als textexterne Komponenten die Situation, die Textfunktion und die Unterscheidung in fachsprachliche und nicht-fachsprachliche Texte. Zusammen mit den Wort- und Satzbedeutungen im Text macht diese Komponente den übersummativen Textsinn aus. Die Kategorie erscheint uns inhomogen und z.T. überflüssig. Wenn die Unterscheidung zwischen Rezeptions- und Produktionskategorien aufgehoben werden würde, könnten unter der Kategorie PRAGMATIK große Teile der hier behandelten Faktoren untersucht werden.

Homogener ist die Kategorie SEMANTIK, die primär mit dem Instrumentarium der strukturellen Semantik (Seme, Isotopieketten, Wortfelder) den Text untersucht. Hierhin gehören auch Konnotationen und Assoziationen, die mit den Wörtern verbunden sind. Die Kategorie LEXIK beschäftigt sich mit fachsprachlicher Lexik, Terminologie und Phraseologie (und ist damit stark mit der Kategorie THEMATIK und auch der Kategorie PRAGMATIK verbunden).

In der Kategorie PRAGMATIK werden in Abhängigkeit von der Sender-Empfänger-Relation Übersetzungszweck, kulturelle und sozi- 
olektale Unterschiede behandelt, und schließlich werden unter STILISTIK text-sorten- und funktionsbedingte Formulierungsbeschränkungen und die Möglichkeit, von der Sprachnorm abzuweichen, behandelt.

Ein Hauptproblem der Arbeit von stolze ist wie schon angedeutet die Beurteilung des Stellenwertes, die der Übersetzungszweck für die Übersetzung hat. Der Ansatz ist ein lobenswerter Versuch, eine Rahmentheorie für die Übersetzung aller multiperspektivischen Texte zu schaffen (272). Bei einem solchen Unterfangen müssen unseres Erachtens die beiden Aspekte berücksichtigt werden, die Stolze mit "Treue zur Vorlage" und "Intentionsadäquatheit" umschreibt. Stolze nennt auch bei generellen Aussagen oft beide Aspekte, aber es ist unser Eindruck, daß die Intentionsadäquatheit sowohl bei den praktischen Analysen als auch bei Teilen der Theorie zu kurz kommt. Ein Beispiel haben wir schon bei der Behandlung des Begriffs der "Stimmigkeit" erwähnt. Weiter wird am Anfang des Kapitels über die Kategorie PRAGMATIK zuerst gesagt, daß der Übersetzungszweck nur eine Rahmenbedingung ist, die anderen Kategorien aber nicht außer Kraft setzt. In der Fortsetzung wird dann als Beispiel ein Werbetext behandelt, bei dem der Übersetzungszweck (Erzielen einer ähnlichen Wirkung in der fremden Kultur) tatsächlich die anderen Kategorien teilweise außer Kraft setzt, indem die Stimmigkeit dann nicht die semantischen oder lexikalischen, ja z.T. nicht einmal die thematischen Sinnkomponenten betreffen muß. Unseres Erachtens braucht die Theorie in diesem Punkt eine gewisse Klärung.

Dasselbe gilt für die theoretische Ablehnung von Übersetzungsstrategien, die an Strukturen der Textvorlage gebunden sind. Stolze sagt an mehreren Stellen, daß solche Strategien nur zu schlechten Ergebnissen führen könnten (z.B. 73: "Wer so mechanisch übersetzt, produziert die notorischen Sekundärtexte, denen man das Übersetztsein schon von weitem ansieht."). Bei gewissen Textsorten und bestimmten Übersetzungsaufgaben räumt sie aber der "Quasi-Selbststeuerung des Übersetzungsprozesses" (Wills, zitiert S. 73) eine Berechtigung ein. In einem Kapitel über das Übersetzen juristischer Texte (176-190) hält sie bei der Überset-zung von Urteilen die Übernahme der zielsprachlichen Konventionen bei ausgangssprachlichen Gliederungssignalen zu Recht für unerläßlich. Dies liegt im Übersetzungsauftrag begründet (eine soweit möglich auch formal entsprechende Übersetzung des Urteils $\mathrm{zu}$ formulieren). Aber auch bei bestimmten Wortbildungstypen stellt sie die Möglichkeit einer fast automatischen Übersetzung dar (153). Unseres Erachtens würde die Theorie dadurch gewinnen, daß die Möglichkeit der 


\section{4}

Nutzung von text-sortenspezifischen Relationen zwischen sprachlichen Strukturen (die im praktischen Teil der Arbeit weitgehend akzeptiert wird) stärker auch in der Theorie berücksichtigt würde²

Unsere generelle Einschätzung von dieser Arbeit Radegundis Stolzes ist, daß sie sich hier die Sisyphos-Arbeit eines Zusammendenkens von Theorie und Praxis vorgenommen hat, und daß es mit den oben angegebenen Einschränkungen gelungen ist. Die theoretischen Teile sind zu-kunftsweisend (wenn wir auch nicht all ihre Ansichten teilen), aber wünschenswert wäre ein Aufweichen der Haltung gegenüber den nichthermeneutischen Übersetzungstheorien sowie eine analytische Untersuchung davon, was unter "Textsinn" zu verstehen ist.

\section{Literatur}

Engberg, Jan (1992): Signalfunktion und Kodierungsgrad von sprachlichen Merkmalen in Gerichtsurteilen. In: Hermes 9, 65-82.

Gutt, Ernst-August (1991): Translation and Relevance. Oxford/Cambridge: MA: Basil Blackwell..

Reiß, Katharina / Hans J. Vermeer (1984): Grundlegung einer allgemeinen Translationstheorie. Tübingen: Niemeyer.

Wills, Wolfram (1992): Übersetzungsfertigkeit. Annäherung an einen komplexen übersetzungspraktischen Begriff. Tübingen: Gunter Narr.

2 Dabei scheint der von Wills neulich bearbeitete Begriff der Übersetzungsfertigkeit einen möglichen Weg anzuzeigen. Über Fertigkeit sagt er: "Wer über eine Fertigkeit verfügt, hat einen mehr oder minder langwierigen und komplizierten Lernprozeß durchlaufen [...] und sein Verhalten so organisiert, daß es den Charakter eines Routineprozesses annimmt." (Wills 1992, 2). 